\title{
SCHWERPUNKTTHEMA „REPOSITORIEN IN ÖSTERREICH - STATUS QUO UND (ZUKÜNFTIGE) HERAUSFORDERUNGEN“
}

Zusammenfassung: In der Diskussion über die Transformation des wissenschaftlichen Publizierens zeigt sich deutlich, dass Repositorien zukünftig eine noch bedeutsamere Rolle im Zusammenhang mit dem freien Zugang zu Forschungsergebnissen und-daten zukommen könnte und sollte. Daher erschien es uns reizvoll, eine Art Bestandsaufnahme zu „Repositorien in Österreich - Status quo und (zukünftige) Herausforderungen" $z$ initiieren.

Schlüsselwörter: Österreich; Repositorien; Anwenderlnnen; Betreiberlnnen; Status quo; Herausforderungen

\section{SPECIAL ISSUE „REPOSITORIES IN AUSTRIA - STATUS QUO AND (FUTURE) CHALLENGES“}

Abstract: In the debate on the transformation of scholarly publishing, it is getting increasingly obvious that repositories could and should play an even more significant role in the future with regard to an free (open) access to research results and data. It therefore seemed appealing to initiate a kind of survey on "Repositories in Austria status quo and (future) challenges".

Keywords: Austria; Repositories; Users; Operators; Status quo; Challenges

DOI: https://doi.org/10.31263/voebm.v71i1.2041

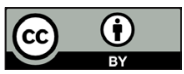

Dieses Werk ist lizenziert unter einer

Creative-Commons-Lizenz Namensnennung 4.0 International 
in der Diskussion über die Transformation des wissenschaftlichen Publizierens zeigt sich deutlich, dass Repositorien zukünftig eine noch bedeutsamere Rolle im Zusammenhang mit dem freien Zugang zu Forschungsergebnissen und -daten zukommen könnte und sollte.

Open Access, Open Data, Open Science, FAIR-Principles und avisierte Initiativen wie die European Open Science Cloud (EOSC) einerseits und die Datenschutzgrundverordnung, rechtliche und ethische Rahmenbedingungen, der Umgang mit sensiblen Daten und die Konkurrenz durch kommerzielle Anbieter auf der anderen Seite. Das ist nur ein Spannungsfeld, dem Repositorien, im Zusammenhang mit ihrer Einbettung in die Wissenschaftslandschaft, laufend ausgesetzt sind. Öffentliche Diskussionen und Veranstaltungen, wie beispielsweise jene, die am 24.10.2017 an der Universität Wien zum Thema „Herausforderungen für Repositorien!?" stattfand, zeigen, dass sich in den letzten Jahren im juristischen, technischen und wissenschaftlichen Bereich einiges geändert hat, auf das Betreiberlnnen von Repositorien möglichst flexibel reagieren müssen, um den ständig steigenden Bedürfnissen seitens der Wissenschaftlerlnnen, Forschungsförderungsorganisationen, etc. gerecht werden zu können.

Daher erschien es uns reizvoll, eine Art Bestandsaufnahme dieses Bereichs zu initiieren. Der Call for Papers für den Themenschwerpunkt „Repositorien in Österreich - Status quo und (zukünftige) Herausforderungen“ stieß auf großes Interesse, das unsere Erwartungen weit übertroffen hat. Mit insgesamt 19 Schwerpunktbeiträgen präsentiert diese Ausgabe eine Vielzahl unterschiedlicher Perspektiven, Themen, Beispiele und Meinungen und erfüllt damit hoffentlich den selbst gestellten Anspruch, die aktuelle österreichische Repositorienlandschaft, aber auch deren Potenziale und Schwierigkeiten sowie den internationalen Kontext abzubilden.

Die vielfältigen Aspekte des Schwerpunktthemas lassen sich kaum in einer „logischen“ Reihenfolge darstellen. Die Herausgeberlnnen haben sich infolgedessen dazu entschlossen, die Beiträge nach der Perspektive aus der sie verfasst wurden (AnwenderInnen und Betreiberlnnen) anzuordnen.

Wir hoffen, dass dieses Schwerpunktheft dazu beiträgt, Repositorien besser kennenzulernen, Möglichkeiten aufzuzeigen, Herausforderungen zu diskutieren und sie für die Zukunft weiterzuentwickeln.

Ihr Redaktionsteam dieser Schwerpunktsausgabe 
Mag. ${ }^{a}$ Dr. ${ }^{\text {in }}$ Susanne Blumesberger, MSc ORCID: http://orcid.org/0000-0001-9018-623X Universität Wien, Bibliotheks- und Archivwesen E-Mail: susanne.blumesberger@univie.ac.at

Mag. Andreas Ferus, MSc ORCID: http://orcid.org/0000-0003-2509-0009 Akademie der bildenden Künste Wien, Universitätsbibliothek E-Mail: a.ferus@akbild.ac.at

Mag. Christian Kaier ORCID: http://orcid.org/0000-0002-8750-6666 Universität Graz, Universitätsbibliothek E-Mail: christian.kaier@uni-graz.at

Mag. . Gertraud Novotny, MSc Wirtschaftsuniversität Wien, Universitätsbibliothek E-Mail: gertraud.novotny@wu.ac.at 